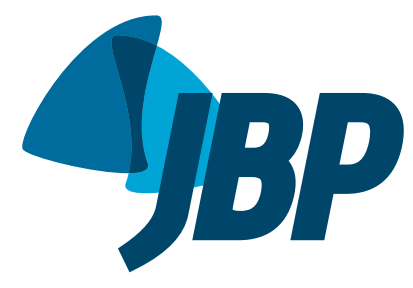

\title{
Effects of the implementation of a hand hygiene education program among ICU professionals: an interrupted time-series analysis
}

\author{
Diana Marcela Prieto Romero,a, Maycon Moura Reboredo ${ }^{1,2, b}$, \\ Edimar Pedrosa Gomes ${ }^{1,2, c}$, Cristina Martins Coelho ${ }^{1, d}$, \\ Maria Aparecida Stroppa de Paula,e, Luciene Carnevale de Souza, ${ }^{1, f}$, \\ Fernando Antonio Basile Colugnati ${ }^{2, \mathrm{~s}}$, Bruno Valle Pinheiro ${ }^{1,2, \mathrm{~h}}$
}

1. Núcleo de Pesquisa em Pneumologia e Terapia Intensiva, Universidade Federal de Juiz de Fora, Juiz de Fora (MG) Brasil.

2. Faculdade de Medicina, Universidade Federal de Juiz de Fora, Juiz de Fora (MG) Brasil.

a. (iD http://orcid.org/0000-0003-1608-8003

b. (D) http://orcid.org/0000-0001-8155-7414

c. (D) http://orcid.org/0000-0002-2135-3628

d. (ID) http://orcid.org/0000-0001-6267-6880

e. (D) http://orcid.org/0000-0003-4665-2402

f. (D) http://orcid.org/0000-0002-8159-9312

g. (D) http://orcid.org/0000-0002-8288-203X

h. (ID http://orcid.org/0000-0002-5288-3533

Submitted: 29 May 2018

Accepted: 23 September 2018

Study carried out at the Hospital

Universitário, Faculdade de Medicina

Universidade Federal de Juiz de Fora, Juiz de Fora (MG) Brasil.

\begin{abstract}
Objective: To evaluate the effects that a hand hygiene education program has on the compliance of health professionals in an ICU. Methods: This was a quasi-experimental study with an interrupted time-series design, conducted over a 12-month period: the 5 months preceding the implementation of a hand hygiene education program (baseline period); the 2 months of the intensive (intervention) phase of the program; and the first 5 months thereafter (post-intervention phase). Hand hygiene compliance was monitored by one of the researchers, unbeknownst to the ICU team. The primary outcome measure was the variation in the rate of hand hygiene compliance. We also evaluated the duration of mechanical ventilation (MV), as well as the incidence of ventilator-associated pneumonia (VAP) at 28 days and 60 days, together with mortality at 28 days and 60 days. Results: On the basis of 959 observations, we found a significant increase in hand hygiene compliance rates-from $31.5 \%$ at baseline to $65.8 \%$ during the intervention phase and $83.8 \%$ during the post-intervention phase, corresponding to prevalence ratios of 2.09 and 2.66, respectively, in comparison with the baseline rate $(p<0.001)$. Despite that improvement, there were no significant changes in duration of MV, VAP incidence (at 28 or 60 days), or mortality (at 28 or 60 days). Conclusions: Our findings indicate that a hand hygiene education program can increase hand hygiene compliance among ICU professionals, although it appears to have no impact on VAP incidence, duration of MV, or mortality
\end{abstract}

Keywords: Hand disinfection; Health personnel; Pneumonia, ventilator-associated; Respiration, artificial; Guideline adherence.

\section{INTRODUCTION}

Healthcare-associated infections (HAIs) are recognized as a major problem among inpatients, prevalence rates of $6.4-8.7 \%$ having been reported in North America and Europe. ${ }^{(1,2)}$ Ventilator-associated pneumonia (VAP) is one of the most common HAIs, being the most common among ICU patients. (3) VAP increases the duration of mechanical ventilation (MV) and the length of hospital stay, resulting in increased costs. ${ }^{(4)}$ Mortality rates are high among patients with VAP, ranging from $20 \%$ to $50 \%$, although it is impossible to determine the extent to which VAP alone accounts for these rates. ${ }^{(5,6)}$

Measures to prevent VAP include the use of noninvasive ventilation in an attempt to avoid endotracheal intubation; the use of protocols to evaluate the possibility of daily sedation interruption and ventilator weaning; keeping the head of the bed elevated; the use of silver nitrate-coated catheters or tubes with increased sealing capacity to allow subglottic secretion drainage; and providing oral care with chlorhexidine, selective oropharyngeal/digestive decontamination, or a combination of the two. $(7,8)$
Although none of the care bundles specifically designed to prevent VAP include proper hand hygiene by health care workers, it is generally recommended for infection prevention, ${ }^{(9)}$ given that transmission of pathogens from one patient to another via the hands of health care workers plays an important role in the pathogenesis of HAIs (including VAP). ${ }^{(10)}$ Although the importance of proper hand hygiene has been established, compliance rates remain low. ${ }^{(11)}$ Studies have shown that educational measures are effective in increasing compliance rates and reducing infection rates. ${ }^{12,13)}$ However, these results are difficult to extrapolate because interventions, observation periods, ICU characteristics, and ICU staff characteristics vary across studies. In addition, few studies have evaluated the impact that a hand hygiene education program alone has on VAP incidence. We hypothesized that such a program might increase hand hygiene compliance and, consequently, reduce the incidence of VAP. To test this hypothesis, we conducted the present study in the ICU of a teaching hospital. 


\section{METHODS}

This was a quasi-experimental study with an interrupted time-series design, conducted between January and December of 2016 in the ICU of the Hospital Universitário da Universidade Federal de Juiz de Fora (HU-UFJF, Federal University of Juiz de Fora University Hospital), located in the city of Juiz de Fora, Brazil. The HU-UFJF is a 150-bed teaching hospital, and the HU-UFJF ICU is a 9-bed medical-surgical ICU for adult patients. The present study was approved by the Research Ethics Committee of the HU-UFJF.

Nosocomial infection control measures already in place in the HU-UFJF ICU included contact isolation (masks, caps, and gowns being worn for contact with patients from whom methicillin-resistant Staphylococcus aureus [MRSA], vancomycin-resistant Enterococcus faecalis, or multidrug-resistant gramnegative bacilli were isolated) and implementation of a VAP prevention bundle including the following: keeping the head of the bed elevated; evaluating the possibility of daily sedation interruption and ventilator weaning; providing oral care with chlorhexidine; and prophylaxis of acute gastroduodenal mucosal injury and deep vein thrombosis.

\section{Procedures}

From June to July of 2016, all health care workers and students in the HU-UFJF ICU participated in a hand hygiene education program. The program consisted of weekly sessions attended by groups of up to eight individuals. During the sessions, the following topics were discussed: the importance of HAIs; the incidence of HAIs in the HU-UFJF ICU; the importance of cross-transmission of HAIs; the role of proper hand hygiene as a preventive measure; and how often health care workers and students in the ICU performed hand hygiene, as well as when they should do it and how to do it correctly. After the first two months, monthly sessions were held from August to December of 2016). During the sessions, the aforementioned topics were discussed again and data on hand hygiene compliance and HAI incidence rates were presented and discussed with participants.

\section{Hand hygiene compliance monitoring}

Hand hygiene compliance was monitored by one of the researchers, unbeknownst to the ICU team. The following hand hygiene practices were surveyed: 1 . hand hygiene before direct contact with patients (even when gloves are worn); 2 . hand hygiene after direct contact with patients (including hand hygiene after glove removal); 3 . hand hygiene before performing aseptic procedures (before handling an invasive device for patient care, with or without gloves); 4. hand hygiene after body fluid exposure risk (after contact with body fluids/excretions, mucous membranes, nonintact skin, or wound dressings and if moving from a contaminated body site to another body site during care of the same patient); and 5. hand hygiene after contact with inanimate surfaces and objects (including medical equipment) in the immediate vicinity of the patient. The observer recorded all hand hygiene opportunities (defined by the presence of one or more of the aforementioned indications for hand hygiene) and whether or not it was performed. Hand hygiene was performed by washing hands with soap and water or by using an alcohol-based hand rub. Compliance rates were calculated by dividing the number of times ICU personnel performed hand hygiene by the number of hand hygiene opportunities. The quality of hand hygiene (performed by washing hands with soap and water or by using an alcohol-based hand rub) was not evaluated. Hand hygiene opportunities were recorded for each staff category, including physicians, nurses, physiotherapists, nursing technicians, students, and other health care workers.

Hand hygiene compliance was monitored by one of the researchers over 2-h periods randomly distributed through the week. Hand hygiene compliance was monitored over a 12-month period: the 5 months preceding the implementation of the hand hygiene education program (baseline period); the 2 months of the intensive (intervention) phase of the program; and the first 5 months thereafter (post-intervention phase).

\section{Outcomes}

The primary outcome measure was the variation in the rate of hand hygiene compliance. A secondary outcome measure was the variation in the rate of hand hygiene compliance by staff category (physicians, nurses, physiotherapists, nursing technicians, students, and other health care workers). We also evaluated the incidence of VAP (expressed as the number of episodes per 1,000 ventilator-days), the proportion of patients who developed VAP, and the duration of MV (number of days off MV over a 28-day period), as well as 28-day and 60-day mortality rates for the baseline period and the post-intervention phase. In patients who had been on MV for at least $48 \mathrm{~h}$, VAP was diagnosed on the basis of a chest X-ray finding of a new or progressive infiltrate, accompanied by at least two of the following findings: fever or hypothermia (i.e., a body temperature above $37.8^{\circ} \mathrm{C}$ or below $36.0^{\circ} \mathrm{C}$ ); leukocytosis or leukopenia (i.e., leukocytes > $12,000 / \mathrm{mm}^{3}$ or $\left.<4,000 / \mathrm{mm}^{3}\right)$; and purulent tracheal secretions, confirmed by semiquantitative culture of tracheal aspirate showing growth of potentially pathogenic bacteria. ${ }^{(4)}$

\section{Statistical analysis}

Data are presented as means and standard deviations, medians and interquartile ranges, or proportions, depending on their characteristics and distribution (Shapiro-Wilk test and visual analysis of the distribution histogram). Between-group differences were assessed by the independent sample t-test, the Mann-Whitney $U$ test, the chi-square test, or Fisher's exact test, as appropriate. Kaplan-Meier analysis was performed in order to compare mortality and VAP incidence at 60 days between the baseline period and 
the post-intervention phase, distribution differences being assessed by the log-rank test.

Generalized estimating equation models adjusted for correlated data were used in order to estimate prevalence ratios, with opportunities $\times$ bed as clusters, a Poisson family distribution, and a log link function. Robust variance estimation was used, an independent correlation structure being assumed. For compliance rates by staff category and for prevalence rates (presented as a graph), the same type of model was used with an interaction term for professionals $x$ observation period (baseline, intervention, and post-intervention). All statistical estimates and tests performed by this model are presented in graph form. All tests were performed with the Statistical Package for the Social Sciences, version 13 (SPSS Inc., Chicago, IL, USA), and MedCalc, version 17.8.6 (MedCalc Software, Mariakerke, Belgium).

\section{RESULTS}

During the study period, 324 patients were admitted to the HU-UFJF ICU: 142 at baseline, 51 during the intervention phase, and 131 during the post-intervention phase. Of those 324 patients, 130 required MV and were therefore at risk of developing VAP: 57 at baseline, 17 during the intervention phase, and 56 during the post-intervention phase. Table 1 shows the baseline demographic and clinical characteristics of the patients on MV admitted before and after the implementation of the hand hygiene education program.

A total of 959 hand hygiene opportunities were observed over the course of 42 2-h periods. Of those 959 opportunities, 419 (43.7\%) were observed before the implementation of the program, $114(11.9 \%)$ were observed during the program, and 426 (44.4\%) were observed after the implementation of the program. Nursing technicians accounted for $41.2 \%$ of all opportunities; physicians, nurses, students, physiotherapists, and other health care workers accounted for $24.5 \%, 14.3 \%, 6.5 \%, 6.3 \%$, and $7.3 \%$, respectively. There was a progressive and significant increase in hand hygiene compliance rates-from $31.5 \%$ at baseline to $65.8 \%$ during the intervention phase and $83.8 \%$ during the post-intervention phase, corresponding to prevalence ratios of 2.09 and 2.66, respectively, in comparison with the baseline rate (Table 2). Although sample size was not calculated, the statistical power of the study was $100 \%$ for a significance level of $5 \%$ and an $84 \%$ increase in compliance. However, this should be interpreted with caution because of the possibility of type II errors (even with a null probability).

The same behavior was observed when we analyzed the rates for each staff category. Post-intervention compliance rates were significantly higher than baseline compliance rates for all health care workers in the ICU except physiotherapists; however, their baseline compliance rates were already high (Figure 1).

There were no significant differences between the baseline period and the post-intervention phase regarding VAP incidence, VAP at 28 days, or VAP at 60 days. In addition, there were no significant differences between the baseline period and the post-intervention phase regarding mortality at 28 days, mortality at 60 days, or the number of days off MV over a 28-day period in the ICU (Table 3). The Kaplan-Meier curves for mortality and VAP incidence showed no differences between the two observation periods (Figure 2).

\section{DISCUSSION}

Our hand hygiene education program resulted in a significant increase in hand hygiene compliance during

Table 1. Baseline and post-intervention characteristics of admitted patients. ${ }^{a}$

\begin{tabular}{lccc}
\multicolumn{1}{c}{ Characteristic } & $\begin{array}{c}\text { Baseline } \\
(\mathbf{n}=\mathbf{5 7})\end{array}$ & $\begin{array}{c}\text { Post-intervention } \\
(\mathbf{n}=\mathbf{5 6})\end{array}$ & $\mathbf{p}$ \\
Age, years & $63.0[28.5]$ & $57.0[17.0]$ & 0.73 \\
Male sex & $31(54.4)$ & $35(62.5)$ & 0.38 \\
SAPS II & $50.3 \pm 19.8$ & $51.9 \pm 18.4$ & 0.65 \\
SOFA & $9[9]$ & $9[5]$ & 0.94 \\
Indication for ICU admission, N (\%) & & & 0.20 \\
$\quad$ Clinical indication & $32(57.1)$ & $26(46.4)$ & \\
Elective surgery & $22(38.6)$ & $26(46.4)$ & \\
Urgent surgery & $3(5.3)$ & $4(7.2)$ & 0.78 \\
Comorbidities & & & 0.24 \\
Hypertension & $27(47.4)$ & $28(50.0)$ & 0.75 \\
Diabetes & $13(22.8)$ & $8(14.3)$ & 0.62 \\
COPD & $7(12.3)$ & $8(14.3)$ & 0.17 \\
Heart failure & $9(15.8)$ & $7(12.5)$ & 0.87 \\
Kidney failure & $6(10.5)$ & $11(19.6)$ & 0.86 \\
Active cancer & $14(24.6)$ & $13(23.2)$ & $12(21.4)$ \\
Immunodeficiency & $13(22.8)$ & 12.5 & \\
\hline
\end{tabular}

SAPS: Simplified Acute Physiology Score; and SOFA: Sequential Organ Failure Assessment. ${ }^{\text {Values expressed as }}$ median [interquartile range], $\mathrm{n}(\%)$, or mean $\pm \mathrm{SD}$. 
the study period. However, it had no impact on VAP incidence, duration of MV, or mortality.

Transmission of pathogens from one patient to another via the hands of health care workers plays an important role in the pathogenesis of HAIs. ${ }^{(14,15)}$ For this reason (and because hand hygiene compliance rates are low), the World Health Organization (WHO) published in 2009 guidelines for implementing and evaluating hand hygiene education programs in health care facilities. ${ }^{(11)}$ The 2009 WHO guidelines recommend five strategies: availability of hand hygiene products at the bedside or for health care professionals to carry with them; educational programs for health care professionals; (verbal and written) reminders in the workplace; performance feedback; and support from the hospital administration in order to encourage staff involvement.

Table 2. Prevalence ratios for hand hygiene compliance during the observation periods.

\begin{tabular}{lccc} 
Observation period & PR & $95 \%$ CI & p \\
Baseline & 1 & & \\
Intervention phase & 2.09 & $2.22-3.19$ & $<0.0001$ \\
Post-intervention phase & 2.66 & $1.52-2.86$ & $<0.0001$ \\
\hline
\end{tabular}

PR: prevalence ratio. Results obtained by a repeatedmeasures Poisson model.
Several studies have evaluated the efficacy of the 2009 WHO guidelines(11) or the effects of some of the guideline components on different outcomes. In 2017, Gould et al. ${ }^{(12)}$ published a systematic review of 26 studies, including randomized and uncontrolled studies, examining the effects of strategies to improve adherence to hand hygiene recommendations. They concluded that programs including all five strategies recommended in the WHO guidelines ${ }^{(11)}$ and those including some but not all of the recommended strategies can increase hand hygiene compliance, the level of evidence being low. Of the studies evaluating programs including all five strategies recommended in the WHO guidelines, only 1 was a randomized study, having shown a $6.3 \%$ difference in hand hygiene compliance between the intervention and control groups. Four other randomized studies evaluated programs including some but not all of the strategies recommended in the WHO guidelines, and a meta-analysis of the results of those studies showed increased hand hygiene compliance in the intervention groups (OR $=1.19 ; 95 \% \mathrm{CI}: 1.01-1.42)$.

In a systematic review employing less stringent inclusion criteria, Luangasanatip et al.(13) found 6 randomized studied and 25 uncontrolled studies. Of the 6 randomized studies, 2 were included in a

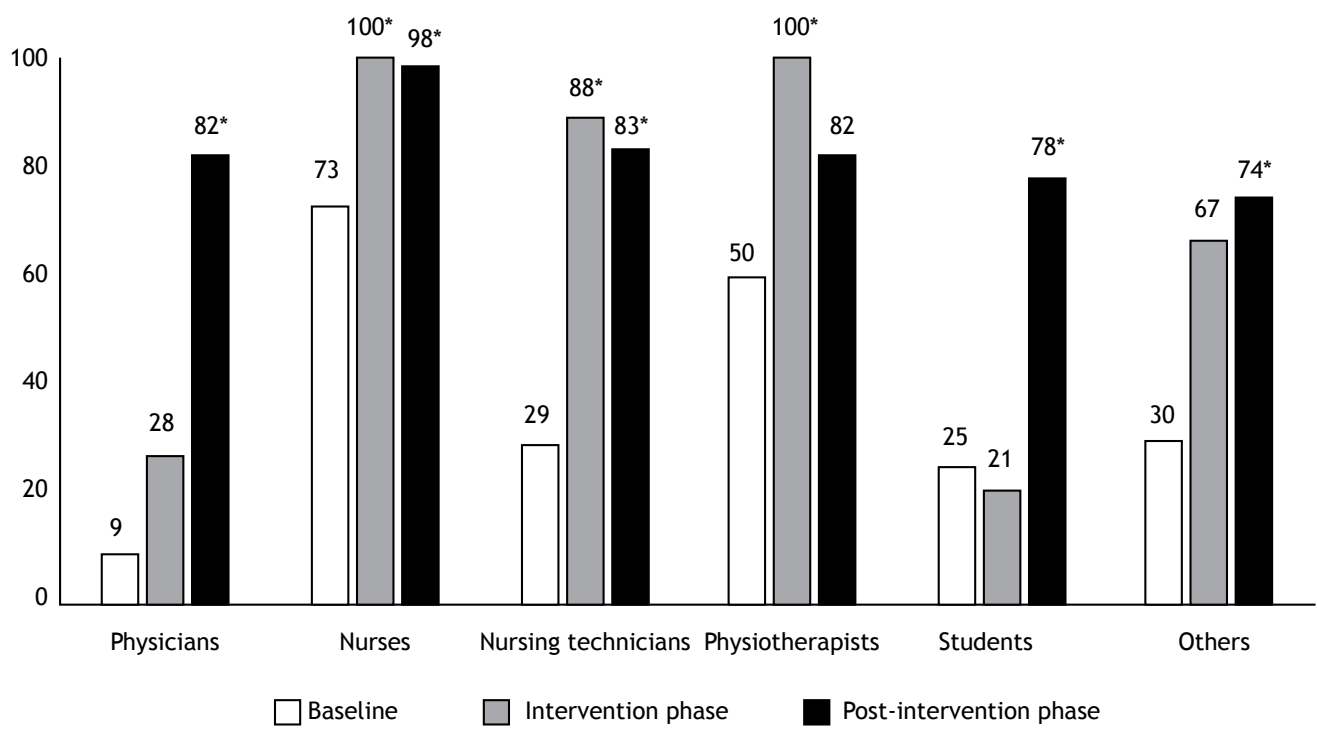

Figure 1. Prevalence rates for hand hygiene compliance in the ICU during the observation periods. ${ }^{*} p<0.05$ vs. baseline.

Table 3. Outcomes for patients on mechanical ventilation. ${ }^{a}$

\begin{tabular}{lccc}
\multicolumn{1}{c}{ Outcome } & $\begin{array}{c}\text { Baseline } \\
(\mathbf{n}=\mathbf{5 7 )}\end{array}$ & $\begin{array}{c}\text { Post-intervention } \\
(\mathbf{n}=\mathbf{5 6})\end{array}$ & $\mathbf{p}$ \\
VAP IDR & 0.011 & 0.012 & 0.39 \\
VAP at 28 days & $4(7.0)$ & $7(12.5)$ & 0.33 \\
VAP at 60 days & $8(14.0)$ & $11(19.6)$ & 0.42 \\
Mortality at 28 days & $31(54.4)$ & $25(44.6)$ & 0.30 \\
Mortality at 60 days & $34(59.6)$ & $33(58.9)$ & 0.94 \\
Days off MV over a 28-day period & $5.9[9.9]$ & $6.1[10.0]$ & 0.94 \\
\hline
\end{tabular}

IDR: incidence density rate (per 1,000 ventilator-days); VAP: ventilator-associated pneumonia; and MV: mechanical ventilation. ${ }^{a}$ Values expressed as absolute value (proportion) or median [interquartile range]. 

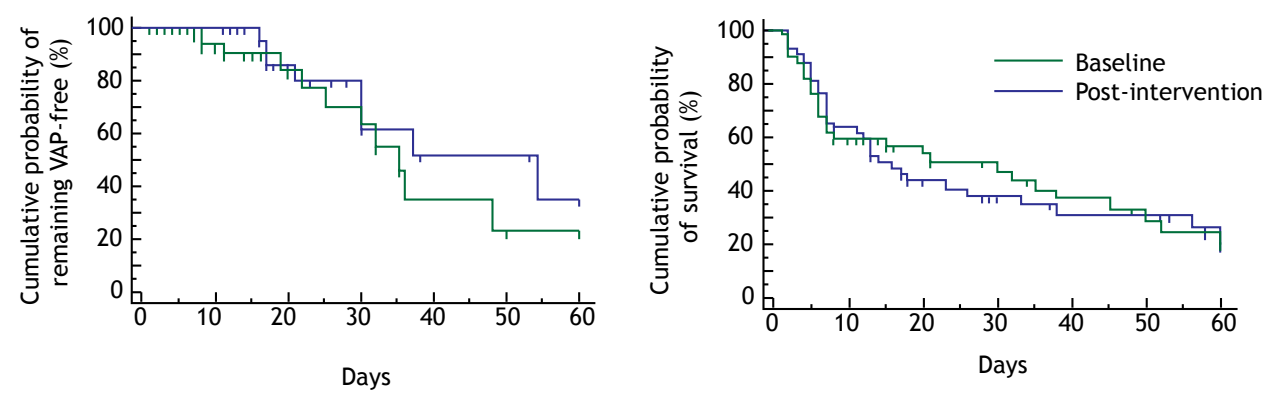

At risk

Baseline

Post-intervention $\quad 56$

Figure 2. Kaplan-Meier curves for ventilator-associated pneumonia (VAP) and mortality at 60 days.

meta-analysis, an OR of 1.35 (95\% CI: $1.04-1.76)$ for adherence to hand hygiene practices having been found in the intervention groups. Of the uncontrolled before-and-after studies, 18 were included in the meta-analysis, which showed a similar result, although with a wider confidence interval (OR $=1.82 ; 95 \%$ CI: 0.2-12.2).

Our study consisted of educational sessions, during which participants received feedback on hand hygiene compliance and ICU infection rates. One of the strategies recommended in the 2009 WHO guidelines, i.e., availability of hand hygiene products at the bedside, had been implemented in our facility before the beginning of the study. The prevalence ratios for hand hygiene compliance during and after the intervention (i.e., 2.09 and 2.66, respectively) in the present study are consistent with those found in previous studies, ${ }^{(16,17)}$ which also consisted of implementing educational measures. In a randomized study conducted over the course of 12 months in 30 ICUs in Canada, implementation of an educational program resulted in an increase in hand hygiene compliance in the intervention and control groups (from $15.8 \%$ to $48.2 \%$ in the former and from $15.9 \%$ to $42.6 \%$ in the latter), corresponding to a $6.3 \%$ difference (95\% CI: 4.3-8.4\%). ${ }^{(16)}$ In a study conducted in 100 hospitals in China and involving a hand hygiene education program, there was an absolute increase of $32.7 \%$ (95\% CI: $15.6-49.7 \%$ ) in the rate of compliance for hand hygiene opportunities before patient contact and of $20.4 \%$ (95\% CI: $5.6-35.2 \%)$ for hand hygiene opportunities after patient contact. ${ }^{(17)}$ It is difficult to compare the aforementioned results because of the specific characteristics of the ICUs evaluated in the studies, including different profiles of patients and health care professionals, with different views of hygiene practices. Therefore, interventions to increase hand hygiene compliance should be tailored to local needs and available resources. In our case, an education-based (and therefore low-cost) intervention was effective in increasing hand hygiene compliance.

Most studies evaluating clinical outcomes of strategies to increase hand hygiene compliance have found reductions in MRSA infection and colonization rates, as well as a reduction in Clostridium difficile infections. (18-20) Few studies have evaluated the effects of such strategies on VAP incidence. In one such study, ${ }^{(21)}$ conducted in two cardiovascular surgery ICUs, an educational program combined with hand hygiene compliance monitoring and oral care resulted in a $59 \%$ reduction in VAP incidence. Although it was impossible to establish the individual impact of each measure (hand hygiene compliance monitoring and oral care), the authors found a negative correlation between hand hygiene compliance and VAP incidence $\left(r^{2}=0.878\right.$; $\mathrm{p}<0.001$ ), a finding that suggests the importance of hand hygiene compliance. ${ }^{(21)}$ In another study, ${ }^{(22)}$ a program to increase hand hygiene compliance was implemented in 150 inpatient units in 12 hospitals. In addition to increasing compliance rates (from $58.1 \%$ to $94.7 \%$ over the course of two years), the program reduced the incidence of VAP (from $49 \%$ to $45 \%$; $p=$ 0.045). ${ }^{(22)}$ Likewise, a 12-month educational program conducted in the ICU of a tertiary hospital resulted in a $75 \%$ increase in hand hygiene compliance rates and a reduction in VAP incidence (from 6.9 episodes per 1,000 ventilator-days to 3.7 episodes per 1,000 ventilator-days; $\mathrm{p}<0.01)$. $^{(23)}$

In our study, we found no association between improved hand hygiene compliance and reduced VAP incidence. This might be due to the low incidence of MRSA VAP in our ICU, hand hygiene being most effective in reducing MRSA infections. Given that gram-negative bacteria (particularly Pseudomonas aeruginosa and Acinetobacter baumannii) are the most common causes of VAP in our ICU, improved hand hygiene compliance might have had no impact on VAP incidence. Our study has limitations that might explain why improved hand hygiene compliance had no impact on VAP incidence. First, the magnitude of improvement might have been insufficient to reduce VAP incidence. Second, the periods of hand hygiene compliance monitoring and the number of patients observed might have been insufficient, resulting in limited statistical power to detect the clinical effect. Third, the observer recorded whether or not hand hygiene was performed; hand hygiene 
technique was not recorded. Finally, because VAP is multifactorial, the fact that only one of the factors involved in its pathogenesis was addressed might have been insufficient to reduce VAP incidence. In addition to having had no impact on VAP incidence, improved hand hygiene compliance had no impact on mortality, duration of MV, or length of ICU stay in the present study. This might be due to the fact that our educational program had no impact on VAP incidence, as well as to the fact that the aforementioned outcomes are more closely related to the condition that led to hospitalization than to hospital-acquired infections.

The present study has other limitations that should be considered. Because our study was not a randomized controlled trial, factors other than the educational program itself might have contributed to improved hand hygiene compliance. The ICU team might have noticed the presence of the observer during the periods of hand hygiene compliance monitoring, despite the fact that the observer made an effort to go unnoticed. This might have resulted in improved hand hygiene compliance during the observation periods. Given that the present study was conducted in a single ICU, with its own epidemiological characteristics, the results obtained cannot be necessarily extrapolated to other ICUs.

In conclusion, our findings indicate that a hand hygiene education program can improve hand hygiene compliance in the ICU, although it appears to have no impact on VAP incidence, mortality, duration of $M V$, or length of ICU stay.

\section{REFERENCES}

1. Zarb P, Coignard B, Griskeviciene J, Muller A, Vankerckhoven V Weist $K$, et al. The European Centre for Disease Prevention and Control (ECDC) pilot point prevalence survey of healthcare-associated infections and antimicrobial use. Euro Surveill. 2012;17(46). pii: 20316.

2. Rutledge-Taylor K, Matlow A, Gravel D, Embree J, Le Saux N Johnston $L$, et al. A point prevalence survey of health care-associated infections in Canadian pediatric inpatients. Am J Infect Control. 2012;40(6):491-6. https://doi.org/10.1016/j.ajic.2011.08.008

3. Magill SS, Edwards JR, Fridkin SK; Emerging Infections program Healthcare-Associated Infections and Antimicrobial Use Prevalence Survey Team. Survey of health care-associated infections. N Engl J Med. 2014;370(26):2542-3. https://doi.org/10.1056/NEJMc1405194

4. Kalil AC, Metersky ML, Klompas M, Muscedere J, Sweeney DA Palmer LB, et al. Management of Adults With Hospital-acquired and Ventilator-associated Pneumonia: 2016 Clinical Practice Guidelines by the Infectious Diseases Society of America and the American Thoracic Society. Clin Infect Dis. 2016;63(5):e61-e111.

5. Melsen WG, Rovers MM, Groenwold RH, Bergmans DC, Camus $\mathrm{C}$, Bauer TT, et al. Attributable mortality of ventilator-associated pneumonia: a meta-analysis of individual patient data from randomised prevention studies. Lancet Infect Dis. 2013;13(8):66571.

6. Bekaert $M$, Timsit JF, Vansteelandt $S$, Depuydt $P$, Vésin $A$ Garrouste-Orgeas $M$, et al. Attributable mortality of ventilatorassociated pneumonia: a reappraisal using causal analysis. Am $J$ Respir Crit Care Med. 2011;184(10):1133-9. https://doi.org/10.1164/ rccm.201105-08670C

7. Klompas M, Branson R, Eichenwald EC, Greene LR, Howell MD Lee $G$, et al. Strategies to prevent ventilator-associated pneumonia in acute care hospitals: 2014 update. Infect Control Hosp Epidemiol. 2014;35 Suppl 2:S133-54.

8. Rello J, Afonso E, Lisboa T, Ricart M, Balsera B, Rovira A, et al. A care bundle approach for prevention of ventilator-associated pneumonia. Clin Microbiol Infect. 2013;19(4):363-9. https://doi.org/10.1111/ j.1469-0691.2012.03808.x

9. Boyce JM, Pittet D, Healthcare Infection Control Practices Advisory Committee, HICPAC/SHEA/APIC/IDSA Hand hygiene Task Force. Guideline for Hand Hygiene in Health-Care Settings. Recommendations of the Healthcare Infection Control Practices Advisory Committee and the HICPAC/SHEA/APIC/IDSA Hand Hygiene Task Force. Society for Healthcare Epidemiology of America/Association for Professionals in Infection Control/Infectious Diseases Society of America. MMWR Recomm Rep. 2002;51(RR16):1-45, quiz CE1-4

10. Strich JR, Palmore TN. Preventing Transmission of Multidrug Resistant Pathogens in the Intensive Care Unit. Infect Dis Clin North Am. 2017;31(3):535-50. https://doi.org/10.1016/j.idc.2017.05.010

11. WHO Guidelines on Hand Hygiene in Health Care: First Global Patient Safety Challenge Clean Care Is Safer Care. Geneva: World

Health Organization; 2009.

12. Gould DJ, Moralejo D, Drey N, Chudleigh JH, Taljaard M Interventions to improve hand hygiene compliance in patient care. Cochrane Database Syst Rev. 2010;9:CD005186.

13. Luangasanatip $N$, Hongsuwan $M$, Limmathurotsakul $D$, Lubell $Y$ Lee AS, Harbarth $S$, et al. Comparative efficacy of interventions to promote hand hygiene in hospital: systematic review and network meta-analysis. BMJ. 2015;351:h3728.

14. Pittet $D$. The Lowbury lecture: behaviour in infection control. J Hosp Infect. 2004;58(1):1-13. https://doi.org/10.1016/j.jhin.2004.06.002

15. Teare EL, Cookson B, French $G L$, Jenner EA, Scott $G$, Pallett $A$, et al UK handwashing initiative. J Hosp Infect. 1999;43(1):1-3. https://doi org/10.1053/jhin.1999.0251

16. Mertz D, Dafoe N, Walter SD, Brazil K, Loeb M. Effect of a multifaceted intervention on adherence to hand hygiene among healthcare workers: a cluster-randomized trial. Infect Control Hosp Epidemiol. 2010;31(11):1170-6. https://doi.org/10.1086/656592

17. Huang J, Jiang D, Wang $X$, Liu Y, Fennie K, Burgess J, et al. Changing knowledge, behavior, and practice related to universal precautions among hospital nurses in China. J Contin Educ Nurs. 2002;33(5):21724. https://doi.org/10.3928/0022-0124-20020901-07

18. Stone SP, Fuller C, Savage J, Cookson B, Hayward A, Cooper B, et al. Evaluation of the national Cleanyourhands campaign to reduce Staphylococcus aureus bacteraemia and Clostridium difficile infection in hospitals in England and Wales by improved hand hygiene: four year, prospective, ecological, interrupted time series study. BMJ. 2012;344:e3005

19. Al-Tawfiq JA, Abed MS, Al-Yami N, Birrer RB. Promoting and sustaining a hospital-wide, multifaceted hand hygiene program resulted in significant reduction in health care-associated infections. Am J Infect Control. 2013;41(6):482-6. https://doi.org/10.1016/j. ajic.2012.08.009

20. Kirkland KB, Homa KA, Lasky RA, Ptak JA, Taylor EA, Splaine ME Impact of a hospital-wide hand hygiene initiative on healthcareassociated infections: results of an interrupted time series. BMJ Qual Saf. 2012;21(12):1019-26. https://doi.org/10.1136/ bmjas-2012-000800

21. Su KC, Kou YR, Lin FC, Wu CH, Feng JY, Huang SF, et al. A simplified prevention bundle with dual hand hygiene audit reduces early-onset ventilator-associated pneumonia in cardiovascular surgery units: An interrupted time-series analysis. PLoS One. 2017;12(8):e0182252.

22. Shabot MM, Chassin MR, France AC, Inurria J, Kendrick J, Schmaltz SP. Using the Targeted Solutions Tool® to Improve Hand Hygiene Compliance Is Associated with Decreased Health Care-Associated Infections. Jt Comm J Qual Patient Saf. 2016;42(1):6-17. https://doi. org/10.1016/S1553-7250(16)42001-5

23. Koff MD, Corwin HL, Beach ML, Surgenor SD, Loftus RW Reduction in ventilator associated pneumonia in a mixed intensive care unit after initiation of a novel hand hygiene program. J Crit Care. 2011;26(5):489-495. https://doi.org/10.1016/j.jcrc.2010.12.013 